\title{
EL DISPOSITIVO INTRAUTERINO COMO ANTICONCEPTIVO POST-ABORTO*
}

\author{
David A. Edelman, PhD \\ International Fertility Research Program. - Chapel Hill, North Carolina. \\ Alfredo Goldsmith, MD, MPH \\ International Fertility Research Program. - Chapel Hill, North Carolina.
}

\section{1 - Introducción}

En América Latina el aborto inducido ilegal es uno de los mayores problemas de Salud Pública (1). Por ejemplo, en algunas ciudades colombianas se ha estimado que el número anual de abortos inducidos es casi igual al número de partos de término (2).

Enforzar estrictamente las leyes que prohiben la terminación artificial de un embarazo probablemente no tendría un efecto significativo sobre la incidencia de aborto ilegal. En vez se deben canalizar los esfuerzos para proporcionar educación contraceptiva a las mujeres durante la hospitalización por aborto ilegal inducido. Se pueden iniciar anticonceptivos orales y DIUs después del tratamiento de un aborto (3).

Este trabajo reporta la seguridad y eficacia de la inserción de DIUs inmediatamente después de aborto. Estos datos provienen de las publicaciones de estudios reportados al International Fertility Research Program.

\section{II - Aspectos clínicos}

\section{Retorno de la Fecundidad después del Aborto}

Retardar la inserción de un DIU o la iniciación de otros métodos anticonceptivos eficaces hasta al menos seis semanas después de un aborto expone a la mujer al riesgo de otro embarazo. El promedio para que comience la ovulación después de un aborto efectuado entre las 8-15 semanas de gestación es de dos a tres semanas, y en abortos efectuados a las 16-20 semanas es de cuatro a seis semanas (5). En el 75-90 por ciento de las pacientes, la primera menstruación después del aborto es ovulatoria y continúa ovulatoria en ciclos subsecuentes. Por esto, si métodos eficaces de anticoncepción no fueron iniciados antes de seis semanas después del aborto, el 3 al 6 por ciento de las pacientes podrían quedar embarazadas antes de iniciar la anticon-

* Este trabajo fue realizado en parte gracias a un grant del International Fertility Research Program. Chapel Hill, North Carolina (AID/csd 2979). 
cepción. Esto asume que el Indice Pearl asociado a relaciones no protegidas es de 80 embarazos por cada 100 mujer-años (6).

\section{Seguridad de la Inserción de DIU Posî-Aborto}

La tradición ginecológica ha sido no insertar un DIU en una cavidad uterina infectada $\circ$ potencialmente infectada. Sin embargo, en un estudio doble ciego (7) conducido para evaluar la seguridad de insertar un DIU inmediatamente después de un aborto no se reconocieron diferencias en las tasas de complicación o en la duración de la hospitalización entre pacientes que tuvieron un DIU insertado y aquellas que no lo tuvieron. Fueron evaluadas un total de 2.388 pacientes en las cuales el aborto había sido iniciado antes de la admisión al hospital. Se estimaron que cerca del 80 por ciento de los abortos fueron ilegalmente inducidos.

Las pacientes fueron asignadas a! azar a uno de dos grupos de tratamiento: Lippes Loop $D$ insertado inmediatamente después del tratamiento de aborto (1.179), o ninguna inserción después del tratamiento de aborto ( 1.209 pacientes). No se presentaron diferencias significativas entre los dos grupos de pacientes en relación con los parámetros evaluados incluyendo: duración de la hospitalización (Fig. 1), la temperatura más alta registrada en pacientes que recibieron tratamiento antibiótico (Fig. 2 ), y diferencia en las tasas de complicación que siguen al aborto. En una investigación similar (8) las únicas diferencias estadísticamente significativas entre aquellas pacientes que tuvieron un Lippes Loop D insertado y aquellas que no lo tuvieron, existieron en la duración del primer período menstrual de la paciente y en la cantidad del flujo menstrual que si-

\section{DURACION DE LA HOSPITALIZACION}

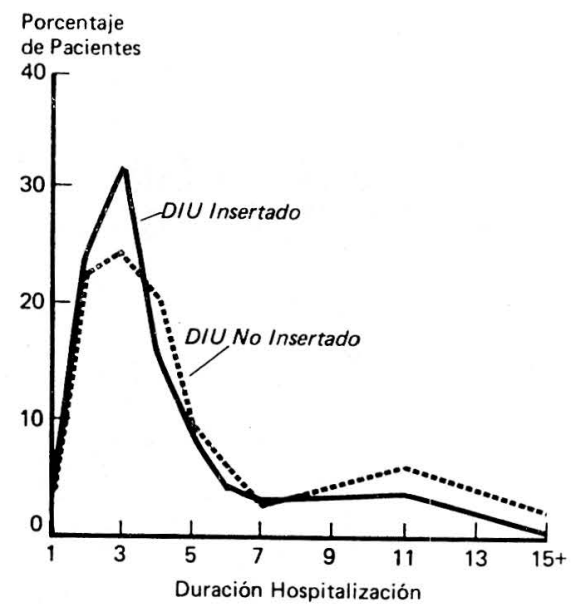

Fuente: Tatum, H. Amer.J.Obstet.Gynec. 112:1,000-1,023, 1972.

TEMPERATURA MAS ALTA REGISTRADA EN PACIENTES CON TRATAMIENTO ANTIBIOTICO

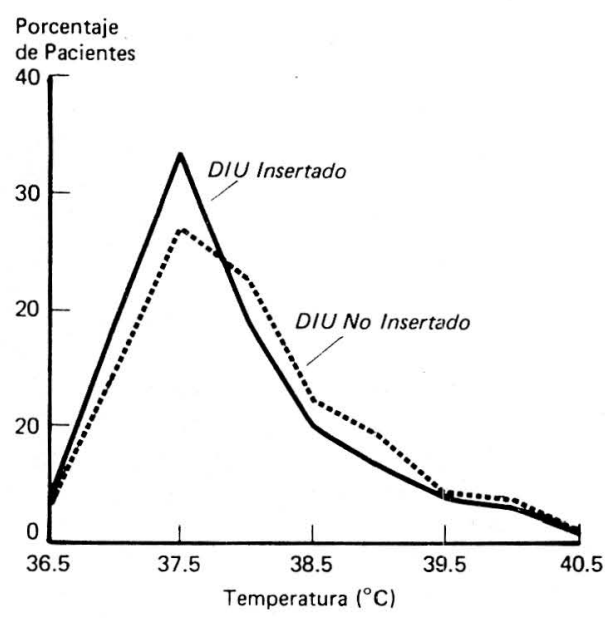

Fuente: Tatum, H. Amer.J.Obstet.Gynec. 112:1,000-1,023, 1972.

guió al tratamiento de aborto por dilatación y curetaje. 
Estos estudios indican que no hay aumento en el riesgo de las pacientes que fueron insertadas con DIU. Al contrario, los riesgos potenciales para estas pacientes no inseriadas con DIU son probablemente más bajos si se consideran también los riesgos asociados con otro embarazo no deseado después del aborto.

\section{Eficacia Anticonceptiva de los DIUs Insertados en el Post-Aborto Inmediato}

La eficacia anticonceptiva de DIUs insertados inmediatamente post-aborto puede ser evaluada usando tasas para eventos pertinentes en la tabla de vida que llevan a la descontinuación en el uso del DIU; embarazo, expulsión y retiro por sangrado $y / 0$ dolor. Mientras que el embarazo y la expulsión son acontecimientos que pueden estar bien definidos $y$ son probablemente independientes a cualquier evento subsecuente a la inserción, el retiro de DIU por razones de sangrado $\mathrm{y} / \mathrm{o}$ dolor no lo es.

Las prácticas de médicos individuales, clínicas $u$ hospitales con respecto al retiro de DIU sin duda afecta la tasa de retiros de DIU por sangrado/ dolor reportada en estudios individuales. El Cuadro I sumariza los resultados de algunos estudios que han reportado sobre la eficacia anticonceptiva de DIUs insertados inmediatamente después de un aborto, ya sea ilegal o legalmente inducido. Las tablas de vida de seis meses o índices Pearl (Cuadro I) indican tasas de embarazos, expulsión y retiro por sangrado $y / 0$ dolor en diferentes DIUs insertados inmediatamente después de aborto. Cuando se compara con la tasa de eventos para inserciones en el intervalo en tipos similares de DIUs (Cuadro II), la inserción de un DIU inmediatamente después de un aborto no lleva a un aumento en las tasas de embarazo, expulsión, o retiro. Por el contrario, existe evidencia que sugiere que las quejas de sangrado y dolor de la paciente son menos frecuentes ch los primeros meses que siguen las inserciones de DIU postaborto cuando se comparan a inserciones de DIU en el intervalo.

\section{III - Comentarios}

Los datos presentados en este trabajo indican que:

a) La inserción de un DIU inmediatamente después de un aborto no resulta en un aumento significativo de las tasas de complicaciones.

b) La eficacia anticonceptiva de los DIUs insertados inmediatamente después de un aborto es similar a la eficacia anticonceptiva de los DIUs insertados durante el período intramenstrual o intermenstrual.

c) Si los métodos anticoncepitvos no se inician antos de seis semanas después de un aborto, al menos del tres al seis por ciento de las mujeres concebirán durante este período de tiempo.

La importancia de usar métodos anticonceptivos eficaces después de un aborto no puede ser suficientemento destacada. La selección de método anticonceptivo de una mujer dependerá de un número de factores que incluyen la accesibilidad y el costo de los anticonceptivos. En situaciones locales en las cuales los anticonceptivos.orales son caros se pueden esperar tasas altas de pérdidas. El objetivo de un programa anticonceptivo en el post-aborto debe ser el proporcionar a las mujeres métodos seguros de anticoncepción para así prevenir embarazos adicionales no deseados, y para promover el uso continuado de estos anticonceptivos. Mientras que el DIU no es el anticonceptivo perfec- 
CUADRO ।

TASAS NETAS ACUMULATIVAS PARA INSERCIONES DE DIU POST-ABORTO A LOS SEIS MESES EN ESTUDIOS SELECCIONADOS

\begin{tabular}{|c|c|c|c|c|c|}
\hline $\begin{array}{l}\text { Lugar y Tipo de } \\
\text { Dispositivo }\end{array}$ & $\begin{array}{l}\text { Primeras } \\
\text { Inserciones }\end{array}$ & $\begin{array}{c}\text { Mujer } \\
\text { Moses/uso }\end{array}$ & Embarazo & $\begin{array}{l}\text { sas de Even } \\
\text { Expulsiones }\end{array}$ & $\begin{array}{l}\text { Retiros por } \\
\text { Sangrado/ } \\
\text { Dolor }\end{array}$ \\
\hline Post aborto inducido & &  & & & \\
\hline $\begin{array}{l}\text { Yugoslavia Dalkon Shield ( } 3 \text { ) } \\
\text { Post aborto inducido }\end{array}$ & 213 & 732 & 0.7 & 0.5 & 0.0 \\
\hline $\begin{array}{l}\text { Salvador Dalkon Shield (9) } \\
\text { Post aborto inducido }\end{array}$ & 259 & 1,284 & 3.5 & 3.7 & 4.2 \\
\hline $\begin{array}{l}\text { Yugoslavia Lippes Loop D ( } 3 \text { ) } \\
\text { Post aborto incompleto }\end{array}$ & 202 & 694 & 1.9 & 3.4 & 3.6 \\
\hline $\begin{array}{l}\text { Chile Lippes Loop D ( } 8) \\
\text { Post aborto incompleto }\end{array}$ & 1470 & - & 0.9 & 10.7 & 6.3 \\
\hline $\begin{array}{l}\text { Chile Membrana Intrauterina (10) } \\
\text { Post aborto inducido }\end{array}$ & 136 & 490 & 0.0 & 3.0 & 0.9 \\
\hline $\begin{array}{l}\text { Yugoslavia Spring Coil ( } 11 \text { ) } \\
\text { * Post aborto inducido }\end{array}$ & 309 & 3,116 & 0.0 & 9.2 & 4.7 \\
\hline $\begin{array}{l}\text { USA Cu-T-200 ( } 12 \text { ) } \\
* \text { Post aborto inducido }\end{array}$ & 140 & 2,216 & 0.0 & 1.1 & 3.8 \\
\hline USA Ypsilon-Y (12) & 194 & 2,692 & 1.3 & 2.2 & 1.8 \\
\hline
\end{tabular}

* Tabulado como Indice Pearl (embarazos/100 años-mujer) y no son comparables con las tasas de tablas de vida de 6 meses.

\section{CUADRO II}

TASAS NETAS CUMULATIVAS A LOS SEIS MESES

INSERCIONES DE DIU EN EL INTERVALO EN ESTUDIOS SELECCIONADOS

\begin{tabular}{|c|c|c|c|c|c|}
\hline $\begin{array}{l}\text { Tipo de } \\
\text { Dispositivo }\end{array}$ & $\begin{array}{l}\text { Primeras } \\
\text { Inserciones }\end{array}$ & $\begin{array}{c}\text { Mujer } \\
\text { Meses/uso }\end{array}$ & Embarazo & sas de Even & $\begin{array}{l}\text { Retiros por } \\
\text { Sangrado/ } \\
\text { Dolor }\end{array}$ \\
\hline $\begin{array}{l}\text { Dalkon Shield, U.K. (13) } \\
\text { Dalkon Shield }\end{array}$ & 3,537 & 20,590 & 1.7 & 1.6 & 2.1 \\
\hline El Salvador (14) & 2,848 & 15,280 & 1.3 & 1.7 & 6.4 \\
\hline $\begin{array}{l}\text { Lippes Loop D, Chile ( } 8 \text { ) } \\
\text { Membrana Intrauterina (15) }\end{array}$ & 14,577 & - & 0.6 & 5.7 & 1.6 \\
\hline Datos Combinados & 119 & 554 & 1.0 & 5.3 & 9.0 \\
\hline Spring Coil (15) & 161 & 684 & 0.0 & 3.5 & 3.6 \\
\hline * Cu-T-200, USA ( 12$)$ & 602 & 10,399 & 1.1 & 1.7 & 3.6 \\
\hline * Ypsilon Y, USA (12) & 492 & 8.610 & 2.4 & 1.7 & 3.1 \\
\hline
\end{tabular}

Tabulado como Indice Pearl (embarazos/100 años-mujer) y no son comparables con las tasas de tablas de vida de 6 meses. 
to $y$ no es el método específicamente seleccionado para todas las mujeres, es barato, proporciona un método efectivo de anticoncepción y puede ser insertado inmediatamente después de un aborto sin aumentar los riesgos para la paciente.

\section{III - Comments}

The date contained in this work indicate that:

a) The insertion of an IUD immediately upon abortion does not result in a significant increase of the rates of complications.

b) The contraceptive efficiency of the IUDs inserted immediately upon a miscarriage is similar to the contraceptive efficiency of IUDs inserted during the intra mnsetrual or intermenstrual period.

c) The contraceptive methods do not begin before six weeks after an abortion, unless three to six percent of the women give birth during this period of time.

The importance of the use of efficient contraceptive methods after an abortion cannot be sufficiently emphasized. The selection of the contraceptive method of a woman depends on a number of factors that include the possibility and the cost of the contraceptive devices. In local situations where oral contraceptives are expensive, higher rates of losses may be expected. The objective program for conrtaception after abortion must be the supply to the women of safe contraceptive methods to prevent additional or unwished pregnancies and the encourage the continued use of such contraceptive devices. While the IUD is not a perfect contracepitve device and is not the method specifically chosen for all women, it is cheap, it offers aneffective methodt for contra- ception and may be inserted immediately after an abortion without increasing risks to the patient.

\section{BIBLIOGRAFIA}

1 ACOSTA, E., DUVA, J. El Aborto Séptico y su Aspecto Económico Social. Revista Colombiana de Obstetricia y Ginecología. Nov. Dic. 1974 .

2 Contraceptive Technology, 1973-1974. The Emory University Family Planning Program, Emory University. Atlanta, Georgia.

3 DELARD, G., TACLA, X., MITRA, M., GOLDSMITH, A., BAEZA, R. Análisis preliminar de la inserción Post-Aborto de un dispositivo intrauterino experimental (Pleated Membrane), Sometido a reproducción.

4 FERGUSON, J. and GOLDSMITH, A. Aborto en América Latina. Presentado en el XI Congreso Colombiano de Obstetricia y Ginecología, diciembre, 1975.

5 GOLDSMITH, A., EDELMAN, D. A., and BRENNER. W. E. Contraception Immediately Post-Abortion. Advances in Planned Parenthood, Vol. X, Proceedings of the Eleventh Anual Meeting of the Association of Planned Parenthood Physicians, Memphis, Tenn., 16-17 April, 1974.

6 GOLDSMITH, A., GOLDBERG, R., EYZAGUI-

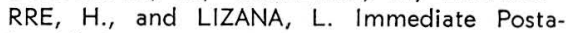
bordal Intrauterine Contraceptive Device Insertion: A Double-Blind Study. Am. J. Obstet. Gynecol. 112:957, 1972.

7 LAMPE, L., RANDIC, L., THOMAS, M., KESSEL, E. One-Year Evaluation of the Spring Coil IUD in Debrecen, Hungary and Rijeka, Yugoslavia. Presented at the VIII World Congress on Fertility and Sterility, Buenos Aires, Argentina, November 3-9, 1974.

8 LAUERSON, N. H., CEDERQVIST, L. L., DONOVAN, S., and FUCHS, F. Comparison of Three Intrauterine Contraceptive Devices: The Antigon- $F$, The Ypsilon- $Y$, and The Copper T-200. Fertil. Steril. 26: 638, 1975.

9 MADRIGAL, V.. THOMAS, M., GOLDSMITH, A., and EDELMAN, D. A. A Two Year Evaluation of the Dalkon Shield in San Salvador. Presented at the VIII World Congress o nFertility and Sterility, Buenos Aires, Arwas gentina, November, 1974.

10 QUAN, A., EDELMAN, D. A., GOLDSMITH, A ZAPPALA-BADIA, D., and THOMAS, M. N. 
Immediate Post-Abortion Insertion of the Dalkon Shield. Contraception. 12: 1, 1975.

11 SNOWDEN, R. and WILLIAMS, M. The United Kingdom Field Trial of the Dalkon

Shield (Second Report). Report of the Family Planning Research Unit. The University of Exeter, Devon, England, 1974.

12 TATUM, H. J. Intrauterine Contraception. Am. J. Obstet. Gynecol. 112: 1.000, 1972.
Rev. Col. Obst. y Ginec.

13 TIETZE, C. and LEWIT, S. Use-Effectiveness of Oral and Intrauterine Contraception. Fertil. Steril. 22 : 508, 1971.

14 VORHERR, H. Contraception After Abortion and Post-Partum. Am. J. Obstet. Gynecol. 117: 1002, 1973.

15 Datos sin publicar del International Fertility Research Program. 